> Depuis les travaux de Johnson et North, il est admis que les opiacés augmentent l'activité des neurones dopaminergiques par un mécanisme de désinhibition impliquant des neurones GABAergiques. Des données récentes appellent cependant à une mise à jour de ce modèle. Ainsi, les neurones GABAergiques par lesquels les opiacés exercent leur action sont maintenant localisés anatomiquement de manière plus précise et caractérisés fonctionnellement. Une structure cérébrale, découverte cette dernière décennie, en continuité avec la partie postérieure de l'aire tegmentale ventrale, est en effet le substrat neuroanatomique de la désinhibition GABAergique. En outre, d'autres données mettent aussi en évidence le rôle essentiel de la transmission glutamatergique dans cette régulation opioïdergique des neurones dopaminergiques. Lors d'un sevrage prolongé aux opiacés, la balance entre les entrées inhibitrices et excitatrices sur les neurones dopaminergiques se trouve perturbée. Ces résultats amènent ainsi à proposer une hypothèse originale pour expliquer les conséquences d'un sevrage prolongé aux opiacés sur l'humeur. <

Les opiacés, dont la morphine, ont des propriétés analgésiques et addictives dues à la stimulation des récepteurs de type $\mu^{1}[1]$. Sans en être le seul substrat, les systèmes dopaminergiques jouent un rôle important dans la dépendance aux opiacés et, depuis 1992 et les travaux fondateurs de Johnson et North [2], un modèle dit de désinhibition a été décrit afin d'expliquer ce recrutement des systèmes dopaminergiques mésencéphaliques. Dans ce modèle, les opiacés

Vignette (Photo @ Sébastien Granier).

${ }^{1}$ II existe trois types de récepteurs des opiacés : mu $(\mu)$, delta $(\delta)$ et kappa $(\kappa)$, distribués dans le système nerveux. Ces récepteurs modulent plusieurs fonctions, dont la réponse à la douleur, au stress et le contrôle des émotions. Ces récepteurs sont une classe de récepteurs couplés aux protéines $G$.

\section{Impact des opiacés sur les neurones dopaminergiques}

Jennifer Kaufling ${ }^{1}$, Marie-José Freund-Mercier ${ }^{2,3}$, Michel Barrot ${ }^{2}$

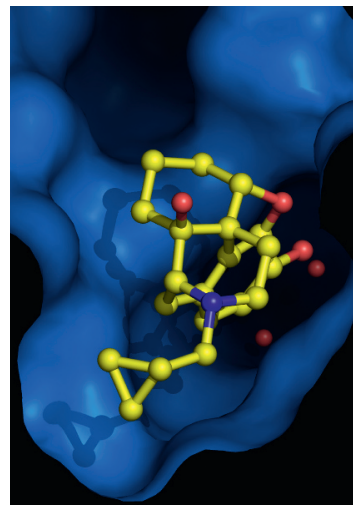

${ }^{1}$ MRC Brain network dynamics unit, université d'Oxford, Mansfield road, Oxford OXI 3TH, Royaume-Uni ;

${ }^{2}$ Institut des neurosciences cellulaires et intégratives, UPR3212 CNRS, 5, rue Blaise Pascal, 67084 Strasbourg, France;

${ }^{3}$ Université de Strasbourg, France. jennifer.kaufling@

excitent indirectement les neurones pharm.ox.ac.uk

dopaminergiques de l'aire tegmentale ventrale (VTA, pour ventral tegmental area) en levant un frein inhibiteur exercé localement par des neurones GABAergiques (produisant de l'acide $\gamma$-aminobutyrique, GABA). Si, près d'un quart de siècle plus tard, le modèle est toujours d'actualité dans ses grandes lignes, certaines mises à jour sont aujourd'hui indispensables. En effet, alors qu'initialement des inter-neurones de la VTA étaient supposés être à l'origine de la désinhibition, le rôle d'une autre population neuronale GABAergique, postérieure à la VTA $[3,4]$, de même que celui de la transmission glutamatergique dans la VTA, apparaissent maintenant évidents [4].

\section{La queue de la VTA, un nouveau site d'action des opiacés}

Évènement de plus en plus rare en neurosciences, une nouvelle structure cérébrale a été découverte et décrite dans la dernière décennie. Située dans le prolongement postérieur de la VTA (Figure IA), elle a été nommée queue de l'aire tegmentale ventrale (tVTA, pour tail of the VTA) [5, 6] ou noyau rostromédian du tegmentum (RMTg, pour rostromedial tegmental nucleus) [7] par les équipes pionnières qui l'ont décrite chez le rat. Depuis, sur la base de son connectome ${ }^{2}$, l'équivalent de cette structure a été observé chez la souris [8] et chez le singe [9], mais sa description chez l'homme reste encore à réaliser. Principalement GABAergique, la tVTA innerve massivement les neurones dopaminergiques de la VTA mais aussi de la substance noire compacte ${ }^{3}$

\footnotetext{
${ }^{2}$ Carte de ses différentes connexions.
}

${ }^{3}$ La substance noire est une petite structure cérébrale qui comporte seulement quatre cent mille neurones dont les corps cellulaires envoient des prolongements en direction des neurones d'un gros noyau cérébral, le striatum. Le neurotransmetteur qui intervient dans ces synapses est la dopamine, présente en grande quantité dans la substance noire et dans le striatum. 


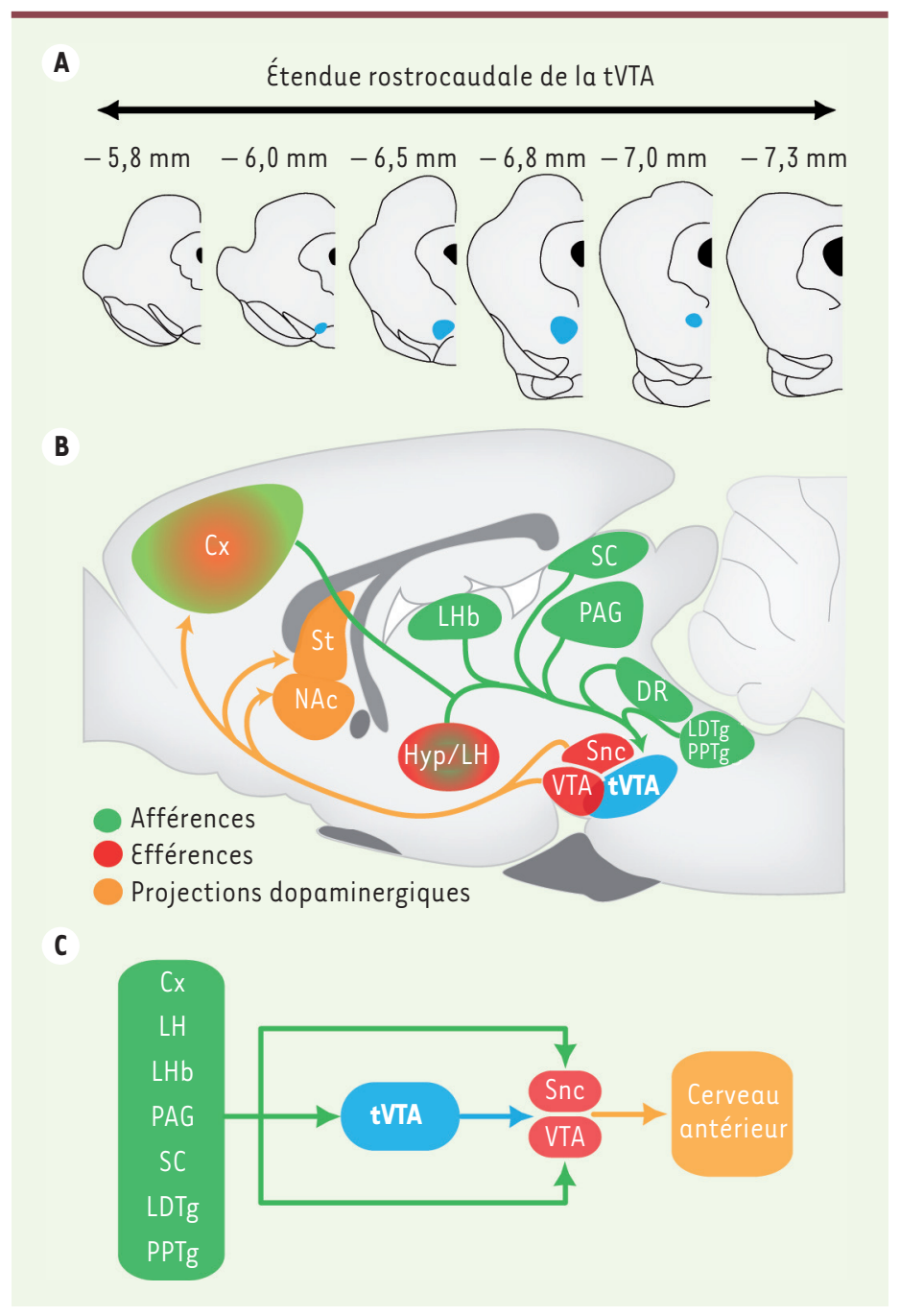

$[7,10]$ (Figures 1B, 1C). La tVTA est également l'efférence $e^{4}$ principale de l'habénula latérale ${ }^{5}[6$, $7]$, qui contribue vraisemblablement aux effets aversifs de la cocaïne [11] et à l'état de type $(\rightarrow)$ Voir la Nouvelle de K. Valentinova et al., $m / s n^{\circ} 5$, mai 2015, page 478 dépressif accompagnant son sevrage $[12,13](\rightarrow)$.

La mise en évidence du rôle de la tVTA dans la réponse dopaminergique aux opiacés a aussi constitué une avancée majeure de ces dernières années [4]. Des expériences conduites chez le rat anesthésié ont en effet montré que l'augmentation de la fréquence de décharge (c'est-à-dire de la fréquence d'émission de potentiels d'action) des neurones dopaminergiques de la VTA, sous l'effet des opiacés, est essentiellement liée à l'inhibition des neurones GABAergiques de la tVTA (Figures 2 et 3A).

Les récepteurs des opioïdes sont exprimés dans l'ensemble du système nerveux [14]. Si la distribution globale des récepteurs de type $\mu$ est

${ }^{4}$ Une efférence est une structure vers laquelle une région cérébrale considérée projette (exemple : la VTA et la substance noire compacte sont des efférences de la tVTA). Une afférence est au contraire une structure dont les axones arrivent et font synapse dans la région considérée (exemple : la tVTA est une afférence de la VTA et de la substance noire compacte).

${ }^{5}$ L'habénula appartient à l'épithalamus et se compose de chaque côté de deux noyaux médian et latéral.

Figure 1. Localisation et connectivité de la tVTA. A. Représentation graphique de coupes coronales de cerveau de rat organisées selon l'axe rostrocaudal (la distance au bregma est donnée en $\mathrm{mm}$ ). La localisation de la tVTA est représentée par des cercles bleus. La tVTA s'étend de $-6 \mathrm{~mm}$ à $-7 \mathrm{~mm}$ par rapport au Bregma. B. Représentation schématique d'une coupe sagittale médiane de cerveau de rat. Les afférences à la tVTA sont nombreuses et étendues. Les plus importantes sont représentées en vert. Les efférences de la tVTA sont plus limitées. Elles concernent les systèmes dopaminergiques mésencéphaliques et l'aire hypothalamique latérale. Elles sont représentées en rouge. Enfin, en orange, on observe les structures cibles des projections dopaminergiques. C. Schéma illustrant la place de la tVTA au sein de l'encéphale. $C_{x}$ : cortex ; DR : noyau du raphé dorsal ; Hyp : hypothalamus ; LDTg : noyau tegmental latérodorsal ; LH : aire hypothalamique latérale; $L H b$ : habénula latérale; PAG : substance grise périaqueducale ; NAc : noyau accumbens; PPTg : noyau tegmental pédonculopontin ; SC : collicules supérieurs ; SNc : substance noire compacte ; St : striatum ; tVTA : queue de la VTA; VTA : aire tegmentale ventrale.

semblable entre les espèces de mammifères, il existe néanmoins quelques différences interspécifiques dans cette distribution ainsi que dans les niveaux d'expression entre les structures cérébrales [15-18]. Ces récepteurs sont toutefois présents, à des niveaux modérés, dans la VTA de tous les mammifères qui ont été étudiés. Dans cette structure, les récepteurs de type $\mu$ sont principalement exprimés par des neurones GABAergiques, mais aussi par une petite fraction de neurones a priori dopaminergiques et/ou glutamatergiques [17, 19]. Ces récepteurs sont également présents sur les terminaisons de certaines afférences de la VTA, aussi bien GABAergiques que glutamatergiques [19]. Chez le rat, l'expression des récepteurs des opioïdes de type $\mu$ est plus forte dans les neurones GABAergiques de la tVTA, tant sur les corps cellulaires que sur les terminaisons de ces neurones au sein de la VTA, qu'elle ne l'est dans les neurones GABAergiques de la VTA elle-même $[4,7]$. Ainsi, in vivo chez le rat anesthésié, la morphine injectée par voie intraveineuse $[20,21]$, ou localement dans la tVTA [4], diminue la fréquence de décharge des neurones de la tVTA. En outre, I'application locale sur des tranches de mésencéphale de morphine, de Met(5)-enképhaline ou d'un agoniste sélectif des récepteurs de type $\mu$, le $\mathrm{DAMGO}^{6}$, hyperpolarise les neurones de la tVTA et réduit leur activité électrique $[20,21]$. Les effets des stimulations électriques ou optogénétiques de la tVTA, qui évoquent (c'est-à-dire génèrent) des courants post-synaptiques inhibiteurs (CPSI) sensibles aux modulateurs des récepteurs de type

\footnotetext{
${ }^{6}\left[\mathrm{D}-\mathrm{Ala}^{2}, \mathrm{NMe}-\mathrm{Phe}^{4}, \mathrm{Gly}-\mathrm{ol} \mathrm{I}^{5}\right]$-enképhaline.
} 


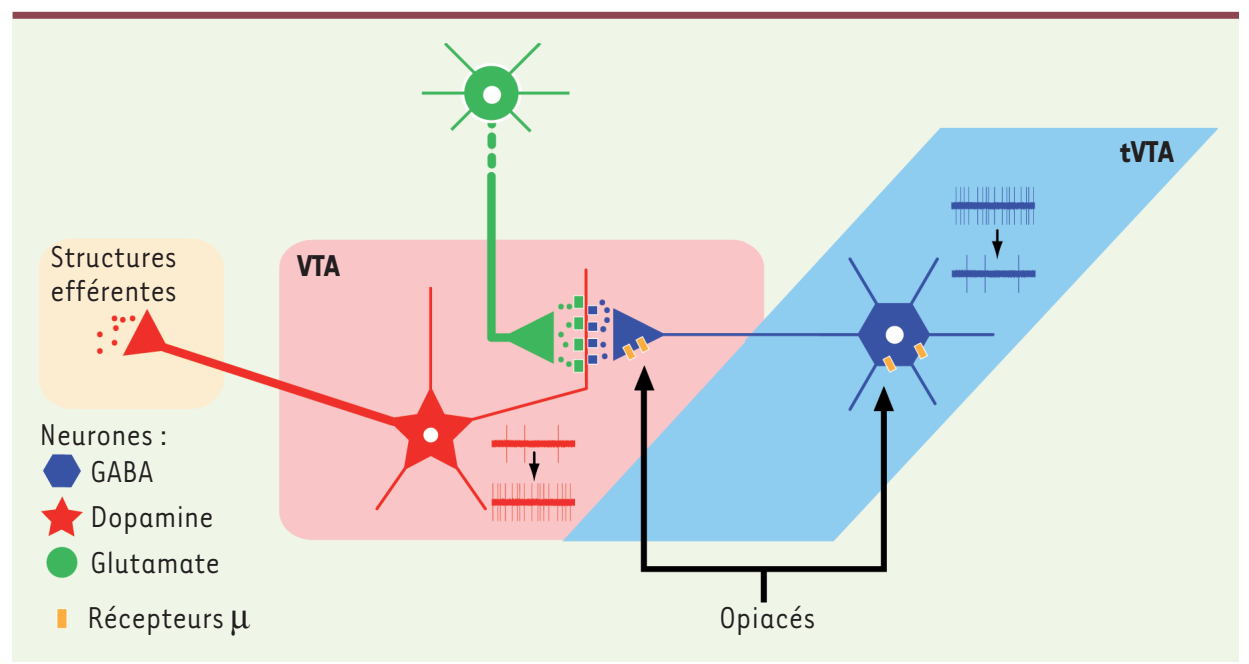

Figure 2. La tVTA comme cible de l'action des opiacés sur les systèmes dopaminergiques. La tVTA exerce un contrôle inhibiteur tonique sur l'activité des neurones dopaminergiques de la VTA. Les opiacés lèvent ce frein via des récepteurs $\mu$ localisés sur les neurones GABA de la tVTA. L'activation des neurones dopaminergiques requiert également un tonus glutamatergique local. tVTA : queue de la VTA; VTA : aire tegmentale ventrale.

$\mu$, suggèrent la présence de ces récepteurs au niveau pré-synaptique, sur les terminaisons GABAergiques de la tVTA qui font synapse sur les neurones dopaminergiques $[22,23]$. Si les CPSI issus d'une stimulation de la tVTA sont très sensibles aux opiacés, l'impact des opiacés est moindre sur les CPSI évoqués par la stimulation des entrées (ou afférences) en provenance du noyau accumbens ou des neurones de la VTA elle-même [23], ce qui renforce l'idée d'un rôle prépondérant joué par la tVTA. Ces données sont en accord avec celles obtenues in vivo, puisque la microinjection de morphine dans la VTA n'induit plus d'augmentation de l'activité électrique des neurones dopaminergiques lorsque les neurones GABAergiques de la tVTA sont inhibés par l'application locale de muscimol, un agoniste des récepteurs $\mathrm{GABA}_{A}{ }^{7}$ [4]. La tVTA est donc la structure clef de la régulation opioïdergique des neurones dopaminergiques.

\section{La transmission glutamatergique, un élément essentiel}

L'activité des neurones dopaminergiques dépend en fait d'une régulation fine entre des entrées inhibitrices GABAergiques et excitatrices glutamatergiques, et l'augmentation d'activité électrique des neurones dopaminergiques de la VTA sous l'effet de la morphine ne peut s'expliquer par sa seule action sur le frein GABAergique que constitue la tVTA. L'innervation glutamatergique des neurones dopaminergiques joue en effet un rôle essentiel dans cette activation par les opiacés (Figure 2), puisque I'application dans la VTA d'antagonistes des récepteurs ionotropes du glutamate empêche l'excitation des neurones dopaminergiques par les opiacés [4]. La stimulation glutamatergique des neurones de la VTA est donc nécessaire pour que leur désinhibition se traduise par une augmentation d'activité des neurones dopaminergiques.

\section{De la morphine chronique au sevrage prolongé}

Les travaux que nous avons décrits ont permis de préciser le mécanisme par lequel la morphine recrute les neurones dopaminergiques.

${ }^{7}$ II existe deux grands types de récepteurs GABAergiques : le récepteur $G_{A B A_{A}}$ et le récepteur $G A B A_{B}$. Le récepteur $G_{A B A_{A}}$ est un membre de la famille des récepteurs-canaux ioniques.
Lorsque l'exposition à la morphine devient chronique, des modifications moléculaires et morphologiques s'opèrent dans les neurones dopaminergiques [24]. Les enregistrements unitaires extracellulaires mettent, quant à eux, en évidence une diminution de la fréquence de décharge des neurones GABAergiques de la tVTA, qui est associée à une augmentation de l'activité de base des neurones dopaminergiques de la VTA [21, 25] et à un déficit dans leur capacité à répondre à une nouvelle exposition à la morphine [25]. Ex vivo, sur des tranches sagittales de cerveau de rats morphinodépendants, l'application d'un bain de morphine a un impact amoindri sur les CPSI GABAergiques des neurones dopaminergiques de la VTA évoqués par la stimulation optogénétique des terminaisons de la tVTA [23]. Lorsque la naloxone, un antagoniste des récepteurs des opioïdes, est appliquée sur ces tranches, elle se révèle aussi moins efficace pour réaugmenter la fréquence de ces CPSI [23]. Ces observations montrent l'existence d'une tolérance partielle des neurones de la tVTA sous morphine chronique. Malgré cette désensibilisation, les récepteurs de type $\mu$ qui restent fonctionnels suffisent in vivo pour exercer encore un contrôle sur l'activité des neurones de la tVTA, puisqu'en enregistrement unitaire, une nouvelle réexposition à la morphine maintient sa capacité à inhiber ces neurones chez l'animal morphino-dépendant [21].

Lors d'un sevrage précipité par l'injection intraveineuse de naltrexone, un antagoniste des récepteurs $\mu$, l'activité électrique des neurones de la tVTA est normalisée, la fréquence de décharge redevenant équivalente à celle enregistrée chez les animaux naïfs [21]. Toutefois, la situation diffère lorsque le sevrage se prolonge [21]. Après 14 jours d'abstinence, l'activité des neurones de la tVTA chez l'animal anesthésié est à nouveau faible, similaire à celle observée chez les animaux morphino- 


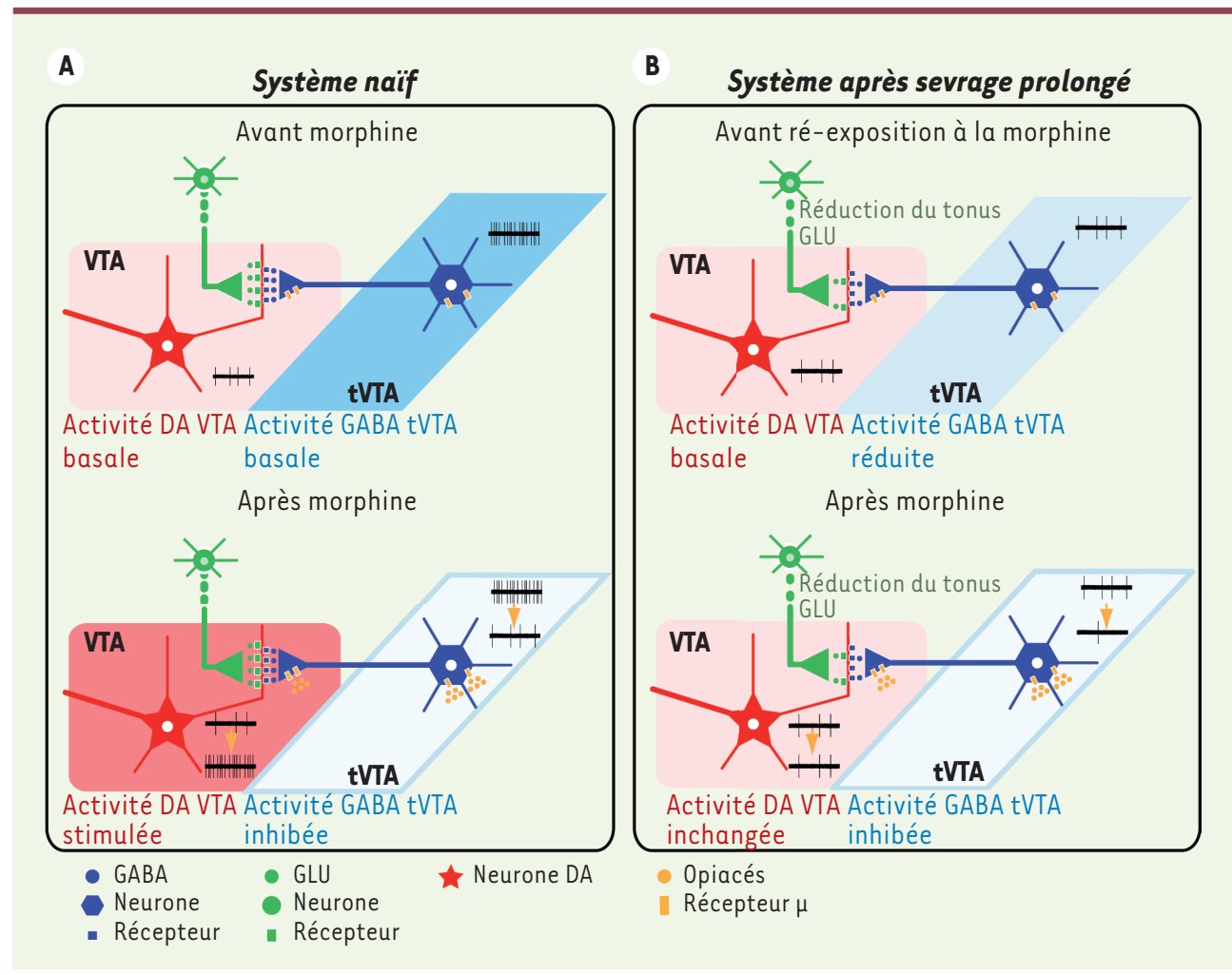

Figure 3. Perte de l'effet des opiacés sur les systèmes dopaminergiques après un sevrage prolongé aux opiacés. $A$. Chez l'animal naïf, la morphine active les neurones dopaminergiques de la VTA via l'inaction du frein tonique exercé par les neurones GABAergiques de la tVTA. B. Chez l'animal en sevrage prolongé, les neurones de la tVTA sont moins actifs que chez les animaux naïfs alors que l'activité des neurones dopaminergiques de la VTA est de retour à un niveau d'activité basale. L'injection aiguë de morphine inhibe toujours les neurones GABAergiques de la tVTA, mais cela reste sans effet sur les neurones dopaminergiques de la VTA. C'est en fait une réduction du tonus glutamatergique sur les neurones dopaminergiques de la VTA qui bloquerait les conséquences excitatrices de la désinhibition des neurones dopaminergiques. DA : dopamine; GLU: glutamate; tVTA : queue de la VTA ; VTA : aire tegmentale ventrale.

dépendants, et la morphine est alors incapable d'activer les neurones dopaminergiques de la VTA $[21,25]$ (Figure 3B). Cette perte de réponse dopaminergique pourrait traduire une tolérance prolongée des récepteurs $\mu$, mais il n'en est rien. En effet, la morphine exerce toujours son effet inhibiteur sur les neurones de la tVTA [21] malgré l'absence de réponse dopaminergique. Il semble donc qu'il y ait une déconnexion entre les neurones GABAergiques de la tVTA et les neurones dopaminergiques de la VTA. Cependant, la stimulation optogénétique des neurones de la tVTA inhibe les neurones dopaminergiques de la VTA de manière similaire chez les animaux naïfs et chez ceux en sevrage prolongé à la morphine. La connexion inhibitrice est donc toujours fonctionnelle entre la tVTA et la VTA !

Le glutamate, comme nous l'avons rappelé, est aussi un élément essentiel dans la réponse des neurones dopaminergiques à la morphine. Afin d'examiner son rôle dans le sevrage prolongé, un mélange d'antagonistes des récepteurs ionotropes du glutamate a été injecté dans la VTA. La conséquence de ce traitement est une réduction de la fréquence de décharge des neurones dopaminergiques chez les animaux naïfs mais pas chez les animaux sevrés depuis deux semaines. Le tonus glutamatergique contrôlant les neurones dopaminergiques de la VTA est donc déficient chez ces animaux en sevrage prolongé. Ce déficit, en perturbant la balance excitation/inhibition (accélérateur/frein) qui régule l'activité des neurones dopaminergiques, apporte une explication à l'absence d'augmentation d'activité des neurones dopaminergiques suite à l'inhibition des neurones GABAergiques de la tVTA par la morphine.

\section{Conclusions}

Les neurones dopaminergiques sont en partie hétérogènes et peuvent coder, via leur activité électrique, diverses informations essentielles aux comportements motivés $^{8}$ et à l'initiation de l'action, qu'il s'agisse de la saillance ${ }^{9}$ d'un stimulus, de sa valeur motivationnelle ${ }^{10}$ ou des changements dans cette valeur $[26,27](\rightarrow)$.

Ainsi, il est connu, depuis les travaux pionniers de Schultz [28],

$(\rightarrow)$ Voir la Synthèse de S. Pichon et $P$. Vuilleumier, $m / s n^{\circ} 8-9$, août-septembre 2011, page 763 que les neurones dopaminergiques du mésencéphale peuvent notamment coder les erreurs de prédiction de récompense ${ }^{11}$. Ce codage neuronal est en général bidirectionnel, mettant en jeu, selon l'information, soit des activations soit des inhibitions de l'activité électrique des neurones dopaminergiques. Chez le rat

\footnotetext{
${ }^{8}$ Comportements dirigés vers un but.

${ }^{9}$ Capacité d'un stimulus à attirer l'attention, que ce soit par ses propriétés propres (par exemple un bruit soudain) ou de par sa valeur pour l'individu (par exemple une cigarette pour un fumeur).

${ }^{10} C^{\prime}$ 'est-à-dire à quel point un individu est motivé pour obtenir ce stimulus ou pas. ${ }^{11}$ II s'agit de la différence entre la valeur prédite de la récompense par l'individu et la valeur réelle obtenue. De nombreuses structures cérébrales semblent coder ce différentiel plutôt que la valeur absolue d'une récompense.
} 
vigile (éveillé, en comportement), les neurones de la tVTA sont, eux, activés de manière phasique par certains stimulus aversifs (déplaisants) alors qu'ils sont inhibés par des stimulus appétitifs (attirants) ou prédictifs d'effets récompensants [29]. Ces réponses sont en fait l'inverse de celles observées pour des neurones dopaminergiques. Cela est en accord avec des données obtenues chez le primate vigile, qui décrivent une structure analogue à la tVTA et qui montrent qu'elle code l'erreur de prédiction de récompense de façon inverse aux neurones dopaminergiques [9]. La question se pose alors d'interpréter, dans ce contexte fonctionnel, les résultats récemment obtenus sur la régulation des neurones dopaminergiques après sevrage morphinique prolongé.

Lors du sevrage prolongé chez le rat, la tVTA conserverait sa capacité à transmettre les informations «négatives/aversives », par une action tonique GABAergique inhibitrice sur les neurones dopaminergiques, mais ne produirait plus d'effet «positif/renforçant », par perte d'efficacité de la désinhibition exercée normalement sur les neurones dopaminergiques. La VTA passerait ainsi d'un mode de fonctionnement à double codage, bidirectionnel, permettant aussi bien une activation qu'une inhibition, à un mode à simple codage, inhibiteur. Cette hypothèse ouvre alors des perspectives aussi bien conceptuelles qu'expérimentales. In fine, ce qui se trouve potentiellement affecté est le codage, l'interprétation et par conséquence l'impact, des évènements de la vie. Bien que cela reste à tester expérimentalement, cette caractéristique fonctionnelle permettrait d'expliquer l'anhédonie, c'est-àdire la perte de plaisir, qui accompagne le sevrage prolongé. La (dé) régulation de l'activité dopaminergique dans la dépression fait actuellement l'objet de nombreux travaux $[30,31](\rightarrow)$ principalement centrés sur le seul niveau d'activité des neurones dopaminergiques. Dans le cadre plus large de l'impact thymique (c'est-à-dire sur l'humeur) du système dopaminergique, les données récentes sur le $\rightarrow$ Voir les Synthèses de G. Sescousse, $m / s$ $n^{\circ}$ 8-9, août-septembre 2015, page 784, et V. Vialou, $m / s n^{\circ} 5$, mai 2013 , page 473 sevrage aux opiacés ouvrent la voie à de nouvelles recherches sur les bases biologiques de l'humeur, qui prendraient cette fois en compte la balance excitatrice/inhibitrice, qui régule l'activité des neurones dopaminergiques, dans le traitement différentiel d'évènements de la vie pouvant être perçus comme positifs ou négatifs. $\diamond$

\section{SUMMARY}

\section{Impact of opiates on dopaminergic neurons}

Since the work of Johnson and North, it is known that opiates increase the activity of dopaminergic neurons by a GABA neuron-mediated desinhibition. This model should however be updated based on recent advances. Thus, the neuroanatomical location of the GABA neurons responsible for this desinhibition has been recently detailed: they belong to a brain structure in continuity with the posterior part of the ventral tegmental area and discovered this past decade. Other data also highlighted the critical role played by glutamatergic transmission in the opioid regulation of dopaminergic neuron activity. During protracted opiate withdrawal, the inhibitory/excitatory balance exerted on dopaminergic neurons is altered. These results are now leading to propose an original hypothesis for explaining the impact of protracted opiate withdrawal on mood. $\diamond$

\section{REMERCIEMENTS}

Ce travail est soutenu par l'ANR-15-CE37-0005.

\section{LIENS D'INTÉRÊT}

Les auteurs déclarent n'avoir aucun lien d'intérêt concernant les données publiées dans cet article.

\section{RÉFÉRENCES}

1. Fields HL, Margolis EB. Understanding opioid reward. Trends Neurosci 2015 ; $38: 217-25$.

2. Johnson SW, North RA. Opioids excite dopamine neurons by hyperpolarization of local interneurons. J Neurosci $1992 ; 12: 483-8$.

3. Bourdy R, Barrot M. A new control center for dopaminergic systems: pulling the VTA by the tail. Trends Neurosci 2012 ; $35: 681-90$.

4. Jalabert M, Bourdy R, Courtin J, et al. Neuronal circuits underlying acute morphine action on dopamine neurons. Proc Natl Acad Sci USA 2011 ; $108: 16446-50$

5. Perrotti LI, Bolaños CA, Choi KH, et al. $\triangle$ FosB accumulates in a GABAergic cell population in the posterior tail of the ventral tegmental area after psychostimulant treatment. EurJ Neurosci 2005 ; 21 : 2817-24.

6. Kaufling J, Veinante P, Pawlowski SA, et al. Afferents to the GABAergic tail of the ventral tegmental area in the rat. J Comp Neurol 2009 ; 513 : 597-621.

7. Jhou TC, Geisler S, Marinelli M, et al. The mesopontine rostromedial tegmental nucleus: A structure targeted by the lateral habenula that projects to the ventral tegmental area of Tsai and substantia nigra compacta. J Comp Neurol 2009 ; 513 : 566-96.

8. Stamatakis AM, Stuber GD. Activation of lateral habenula inputs to the ventral midbrain promotes behavioral avoidance. Nat Neurosci 2012 ; $15: 1105-7$.

9. Hong S, Jhou TC, Smith M, et al. Negative reward signals from the lateral habenula to dopamine neurons are mediated by rostromedial tegmental nucleus in primates. J Neurosci $2011 ; 31: 11457-71$.

10. Kaufling J, Veinante P, Pawlowski SA, et al. $\gamma$-aminobutyric acid cells with cocaine-induced $\triangle F O S B$ in the ventral tegmental area innervate mesolimbic neurons. Biol Psychiatry $2010 ; 67: 88-92$.

11. Jhou TC, Good CH, Rowley CS, et al. Cocaine drives aversive conditioning via delayed activation of dopamine-responsive habenular and midbrain pathways. J Neurosci $2013 ; 33: 7501-12$.

12. Meye FJ, Valentinova K, Lecca S, et al. Cocaine-evoked negative symptoms require AMPA receptor trafficking in the lateral habenula. Nat Neurosci $2015 ; 18: 376-8$

13. Valentinova K, Tchenio A, Meye FJ, et al. L'enfer après le plaisir : contribution de l'habénula latérale aux symptômes dépressifs des drogues. Med Sci (Paris) $2015 ; 31: 478-81$.

14. Le Merrer J, Becker JA, Befort K, et al. Reward processing by the opioid system in the brain. Physiol Rev $2009 ; 89: 1379-412$.

15. Peckys D, Landwehrmeyer GB. Expression of mu, kappa, and delta opioid receptor messenger RNA in the human CNS: a 33P in situ hybridization study. Neuroscience $1999 ; 88$ : 1093-135.

16. Kudo T, Konno K, Uchigashima M, et al. GABAergic neurons in the ventral tegmental area receive dual GABA/enkephalin-mediated inhibitory inputs from the bed nucleus of the stria terminalis. Eur J Neurosci 2014 ; 39 : 1796 809.

17. Ragen BJ, Freeman SM, Laredo SA, et al. $\mu$ and $\kappa$ opioid receptor distribution in the monogamous titi monkey (Callicebus cupreus): implications for social behavior and endocrine functioning. Neuroscience $2015 ; 290: 421-34$.

18. Erbs $\varepsilon$, Faget L, Scherrer G, et al. A mu-delta opioid receptor brain atlas reveals neuronal co-occurrence in subcortical networks. Brain Struct Funct $2015 ; 220: 677-702$

19. Garzón M, Pickel VM. Plasmalemmal mu-opioid receptor distribution mainly in nondopaminergic neurons in the rat ventral tegmental area. Synapse $2001 ; 41: 311-28$

20. Lecca S, Melis M, Luchicchi A, et al. Effects of drugs of abuse on putative rostromedial tegmental neurons, inhibitory afferents to midbrain dopamine cells. Neuropsychopharmacology $2011 ; 36: 589-602$. 


\section{RÉFÉRENCES}

21. Kaufling J, Aston-Jones G. Persistent adaptations in afferents to ventral tegmental dopamine neurons after opiate withdrawal. J Neurosci 2015 ; 35 : 10290-303.

22. Matsui A, Williams JT. Opioid-sensitive GABA inputs from rostromedial tegmental nucleus synapse onto midbrain dopamine neurons. J Neurosci $2011 ; 31$ : 17729-35.

23. Matsui A, Jarvie BC, Robinson BG, et al. Separate GABA afferents to dopamine neurons mediate acute action of opioids, development of tolerance, and expression of withdrawal. Neuron 2014 ; $82: 1346-56$.

24. Mazei-Robison MS, Nestler $\varepsilon$ J. Opiate-induced molecular and cellular plasticity of ventral tegmental area and locus coeruleus catecholamine neurons. Cold Spring Harb Perspect Med 2012 ; 2: $a 012070$.

25. Georges F, Le Moine C, Aston-Jones G. No effect of morphine on ventral tegmental dopamine neurons during withdrawal. J Neurosci $2006 ; 26: 5720-6$.

26. Bromberg-Martin ES, Matsumoto M, Hikosaka 0 . Dopamine in motivational control: rewarding, aversive, and alerting. Neuron $2010 ; 68: 815-34$.
27. Pichon S, Vuilleumier P. Neuro-imagerie et neuroscience des émotions. Med Sci (Paris) $2011 ; 27: 763-70$.

28. Schultz W, Dayan P, Montague PR. A neural substrate of prediction and reward. Science $1997 ; 275$ : 1593-9.

29. Jhou TC, Fields HL, Baxter MG, et al. The rostromedial tegmental nucleus (RMTg), a GABAergic afferent to midbrain dopamine neurons, encodes aversive stimuli and inhibits motor responses. Neuron 2009; 61: 786-800.

30. Sescousse G. Addiction aux jeux d'argent : apport des neurosciences et de la neuro-imagerie. Med Sci (Paris) 2015 ; $31: 784-91$

31. Vialou V. Dépression et regulation de l'activité dopaminergique. Med Sci (Paris) $2013 ; 29: 473-7$

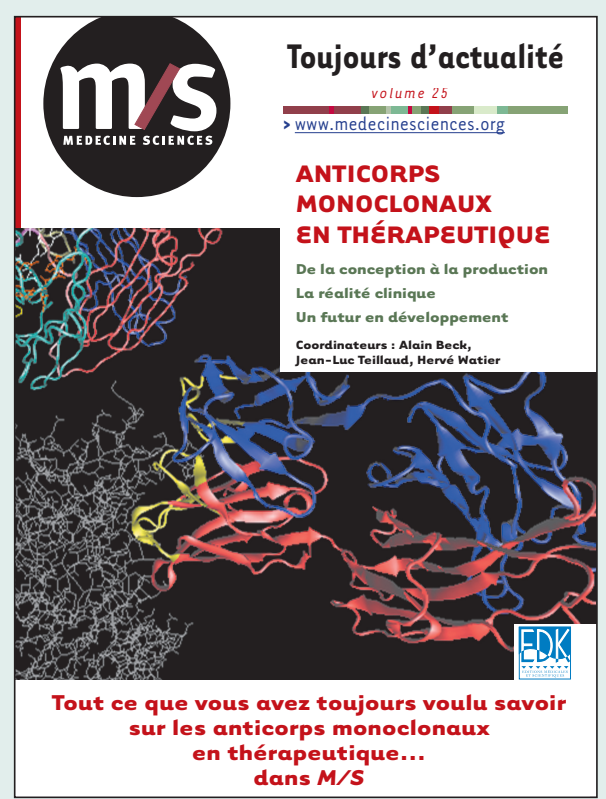

Tout ce que vous avez toujours voulu savoir sur les anticorps monoclonaux en thérapeutique... dans Médecine/Sciences. Pourquoi un numéro spécial de Médecine/Sciences sur les anticorps monoclonaux thérapeutiques? II nous a semblé que le moment était venu de dresser un état des lieux de ces biomédicaments qui prennent désormais une place considérable - et croissante dans les traitements de maladies souvent lourdes et désespérantes. Ce voyage que nous vous proposons à la découverte du monde des anticorps thérapeutiques nous a appris, ou plutôt rappelé, une évidence : les compétences en France sont fortes et nombreuses, qu'elles soient académiques ou industrielles, biotechnologiques ou cliniques. Le paysage français, trop longtemps discret, bruisse désormais de mille initiatives balayant de multiples aspects des anticorps thérapeutiques: études précliniques et cliniques menées avec de nouveaux anticorps dirigés contre des cibles originales, développement de nouveaux formats d'anticorps ou d'anticorps optimisés reposant sur des études structurales et fonctionnelles sophistiquées, recherche active de cibles pertinentes, mise au point de méthodologies de bioproduction, de couplage, etc. L'expansion industrielle rapide de ce champ est un défi que peut et doit relever notre pays, défi tant scientifique qu'économique, avec ses combats pour la propriété intellectuelle et pour l'emploi de nos jeunes scientifiques.

Alain Beck, Jean-Luc Teillaud, Hervé Watier

\section{À retourner à EDK, 109, avenue Aristide Briand, 92541 Montrouge Cedex, France}

Tél. : 0141177405 - Fax : 0149850345 - E-mail : edk@edk.fr

NOM :

Prénom :

Adresse :

Code postal :

Ville :

Pays :

Fonction :

Je souhaite recevoir $\mathrm{M} / \mathrm{S} \mathrm{n}^{\circ} \mathbf{1 2}$ - décembre 2009 (Anticorps monoclonaux en thérapeutique) : $25 €+3 €$ de port $=\mathbf{2 8} € \mathrm{TTC}$ en exemplaire, soit un total de $€$

$\square$ Par chèque, à l'ordre de $\mathbf{E} \mathbf{D} \mathbf{K}$

Par carte bancaire :

Visa

Eurocard/Mastercard

Carte $n^{\circ}$

Date d'expiration :

$\mathrm{N}^{\circ}$ de contrôle au dos de la carte

Signature : 